\title{
In Silico Studies on Fungal Metabolite against Skin Cancer Protein (4,5-Diarylisoxazole HSP90 Chaperone)
}

\author{
Saravanakumar Kandasamy, Sunil Kumar Sahu, and Kathiresan Kandasamy \\ Centre of Advanced Study in Marine Biology, Faculty of Marine Sciences, Annamalai University, Parangipettai 608 502, India \\ Correspondence should be addressed to Kathiresan Kandasamy, kathirsum@rediffmail.com
}

Received 18 June 2012; Accepted 5 August 2012

Academic Editors: M. Clelia and A. R. Ercocen

Copyright (C 2012 Saravanakumar Kandasamy et al. This is an open access article distributed under the Creative Commons Attribution License, which permits unrestricted use, distribution, and reproduction in any medium, provided the original work is properly cited.

\begin{abstract}
This work was to find out the dominant secondary metabolites derived from the fungus Trichoderma and to test them against skin cancer protein. The metabolites were extracted in $80 \%$ methanol from the fungal biomass of Trichoderma isolated from mangrove sediment. The crude methanol extract was purified and analysed for the secondary metabolites by GC-MS. Three predominant compounds (heptadecanoic acid, 16 methyl-, methyl ester; 9,12-octadecadienoic acid; cis-9-octadecenoic acid) identified in the extracts were screened against the skin cancer protein (Hsp90) by in-silico docking method. Of the compounds, heptadecanoic acid, 16 methyl, methyl ester was the most potent having the docking score of $-11.4592 \mathrm{Kcal} / \mathrm{mol}$. This value was better than the standard drug "dyclonine". This work recommends the heptadecanoic acid, 16 methyl, methyl ester for further in vitro and in vivo studies towards its development as anticancer drug.
\end{abstract}

\section{Introduction}

Marine environment is an excellent source of novel compounds with higher pharmaceutical applications [1, 2]. Marine-derived fungus produces a variety of metabolites having enormous biomedical applications. The lowmolecular-weight secondary metabolites are produced by filamentous fungi, plants, algae, bacteria, and animals in response to environmental abiotic and biotic stress [3-5]. Among the fungi, metabolites produced by the Trichoderma species are known to have anticancer activity [6, 7]. Skin cancer is growing as a dreadful human disease as compared to other cancers [8]. However, potent drugs to cure skin cancer are increasingly necessary. Hence, the present study was undertaken to identify the predominant metabolites present in two species of Trichoderma species (Hypocrea spp.) isolated from mangrove sediment and also to test them against skin cancer protein (4,5-diarylisoxazole Hsp90 chaperone) using in-silico molecular docking methods.

\section{Material and Methods}

Two strains of Trichoderma namely Hypocrea lixii TSK8 (JQ809340) and Hypocrea estonica SKS1 (JQ611722) were isolated from mangrove sediment by using Trichoderma selective medium [9] and stored at $4^{\circ} \mathrm{C}$. The two strains were inoculated in a production medium with $\mathrm{pH} 7.2$ and incubated at $28^{\circ} \mathrm{C}$ for 12 days and biomass was then harvested.

2.1. Extraction of the Intra Cellular Secondary Metabolites. The fungal biomass was extracted for intracellular metabolites [10] with some modifications. The fresh biomass was washed three times with sterile distilled water to remove adherent filtrate, and then blotted between folds of sterilized filter paper. The biomass was crushed in a mortar, using $80 \%$ methanol as solvent, and this extraction was repeated three times, and left in separating funnel for 15 min for precipitation. The crude extract was filtered through Whatman No.1 filter paper and the filtrate was dried under vacuum at $40^{\circ} \mathrm{C}$.

2.2. GC-MS Analysis (Gas-Chromatography Mass Spectroscopy). The filtrate was analysed for secondary metabolites by using GCMATE II GC-MS (Agilent). $1 \mu \mathrm{L}$ of the extract was injected through HP-5 capillary column, maintained at the temperature at $220^{\circ} \mathrm{C}$ and Helium as carrier gas. 


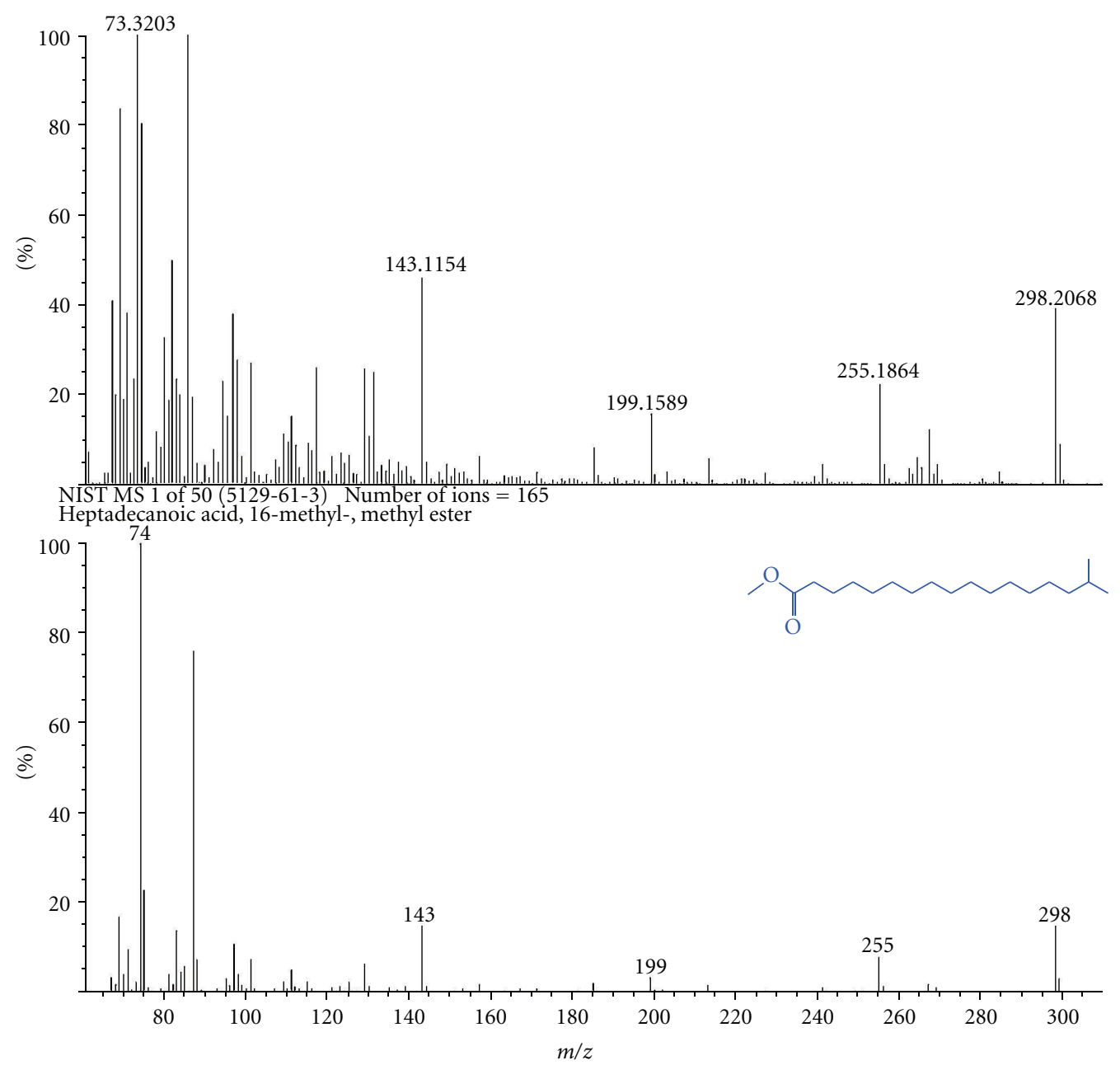

FIgURE 1: GC-MS result of potential compound Heptadecanoic acid, 16 methyl derived from Hypocrea lixii TSK8.

After analysis, the compounds were identified by matching with the known compound library.

2.3. Retrieval of Protein Structure. The target 4,5 diarylisoxazole HSP90 chaperone protein (PDB ID: 2VCJ), having the resolution of $2.0 \AA$, was retrieved from the protein data bank (PDB) (http://www.rcsb.org/pdb/). A standard compound Dyclonine known to have good inhibitory potential against the same skin cancer protein was also docked to compare the effectiveness of the secondary metabolites. Structural and active site studies of the protein were done by using CASTP (Computed Atlas of Surface Topography of Proteins) and Pymol molecular visualization software.

2.4. Compounds Screened. Three compounds, namely, Heptadecanoic acid, 16methyl-, methyl ester; 9,12-Octadecadienoic acid; and cis-9-Octadecenoic acid, identified by GC-MS analysis, were screened against the skin cancer protein. The compound details were retrieved from the Pubchem database and the chemical structures were generated from SMILES notation (simplified molecular input line entry specification) by using the Chemsketch Software (http://www.acdlabs.com).

2.5. Active Site Prediction. Active site of the target protein was predicted by using "Active site prediction tool" from SCFBio Server (http://www.scfbio-iitd.res.in/dock/ActiveSite .jsp) which requires a. pdb file as an input and this tool explains the total number of active sites along with information on their amino acid sequence, cavity points, and the average volume of the cavity.

2.6. Docking Methods . ArgusLab 4.0.1, most common and freely available software, was used for docking analysis. The inhibitor and target protein were geometrically optimized and "Argus dock" docking engine was used. Calculation type was set to "dock" mode whereas "flexible mode" was selected for the ligand. Grid resolution was set to $0.40 \AA$ A. Least energy represented the easy binding character of ligand and receptor.

2.7. Ligand Binding Sites Prediction. After docking the docked structure was saved as ".pdb" file and further explored to predict the binding sites using "Ligand Explorer" software. 
TABLE 1: Docking results of fungal compounds against skin cancer protein.

\begin{tabular}{|c|c|c|c|c|c|}
\hline Compound Name & $\begin{array}{l}\text { Pubchem } \\
\text { ID }\end{array}$ & Compound structure & $\begin{array}{l}\text { Molecular Weight } \\
(\mathrm{g} / \mathrm{mol})\end{array}$ & $\begin{array}{c}\text { Hydrogen } \\
\text { donor/acceptor }\end{array}$ & $\begin{array}{l}\text { Docking Energy } \\
\text { Level (Kcal/mol) }\end{array}$ \\
\hline $\begin{array}{l}\text { Heptadecanoic acid, } 16 \\
\text { methyl, methyl ester }\end{array}$ & 110444 & & 298.50382 & $(0,2)$ & -11.4592 \\
\hline 9,12-Octadecadienoic acid & 5282797 & & 280.445 & $(1,2)$ & -9.286 \\
\hline cis-9-Octadecenoic acid & 445639 & & 282.461 & $(1,2)$ & -8.935 \\
\hline Dyclonine & 3180 & & 289.41248 & $(0,3)$ & -10.088 \\
\hline
\end{tabular}

The predicted binding sites, based on the binding energy, and amino acids make up the binding cavity. Here ligand binding site represents the site where the ligands most efficiently bind with the protein, among all the active sites.

2.8. Drug Likeliness Prediction. Ligand property was predicted by using "Lipinski Drug Filters" (http://www .scfbio-iitd.res.in/utility/LipinskiFilters.jsp). Lipinski rule of five helps in distinguishing drug-like and non-drug-like properties and predicts high probability of success or failure due to drug likeliness for molecules. The Lipsinki filter helps in early preclinical assessment and thereby avoiding costly late-stage preclinical and clinical failures.

\section{Results and Discussion}

The metabolites present in two fungal strains of Hypocrea species were analyzed in GC-MS and the compound (Heptadecanoic acid, 16 methyl) which showed the best result against skin cancer protein is shown in Figure 1. GC-MS result revealed the presence of fatty acids in both the strains. This could be attributed to the fact that polymerisation of acetate results in the formation of a fatty acid or a polyketide [11]. Trichoderma species are already known to produce fatty acids from mussels [11]. They also produce novel cytotoxic compounds such as trichodenone $\mathrm{A}, \mathrm{B}$, and $\mathrm{C}$ which exhibit significant cytotoxicity against leukemia P388 cell line [12, 13]. They have enormous pharmaceutical values such as antibacterial, antiviral [13], antiprotozoal [14], antifungal activities [15], and anti cancer [16].

The protein Hsp90 is abundant in eukaryotic cells and its expression increases when cells are exposed to a variety of stresses [17]. Hsp90 contains three conserved domains: an N-terminal ATP-binding domain, a middle domain, and a carboxy-terminal domain [18]. Hsp90 is upregulated 10fold in tumour cells suggesting that it helps maintaining tumour cell growth and/or survival. Another role for Hsp90 in the maintenance of tumour cells is its ability to inhibit apoptosis [19]. Inhibitors of the Hsp90 molecular chaperone are showing considerable promise as potential chemotherapeutic agents for cancer [20]. Hence, the present work tested the metabolites against 4,5-diarylisoxazole Hsp90 chaperone is a skin cancer protein.

Totally 47 active sites were predicted in the target protein by the "Active site prediction tool". ArgusLab molecular docking software 4.0.1 was used to dock fatty acid compounds against the skin cancer protein (4,5-diarylisoxazole Hsp90 chaperone). The docking interaction of the protein and ligand, and the predicted ligand binding site residues are shown in Figures 2(a) and 2(b), respectively. The docked ligand molecules were selected based on docking energy and good interaction with the active site residues and the results are shown in Table 1. Of three compounds, Heptadecanoic acid, 16 methyl, methyl ester was the most potent having the least docking score of $-11.4592 \mathrm{Kcal} / \mathrm{moL}$. This value was better than that of the potent drug "Dyclonine" [21] which showed the docking score of -10.088 . Lesser the docking score more is the binding capacity of the ligand. Hence, the 


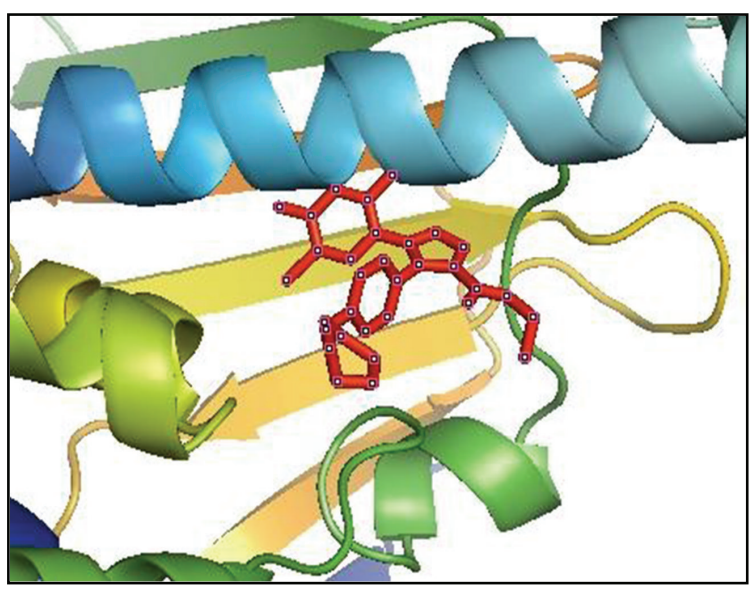

(a)

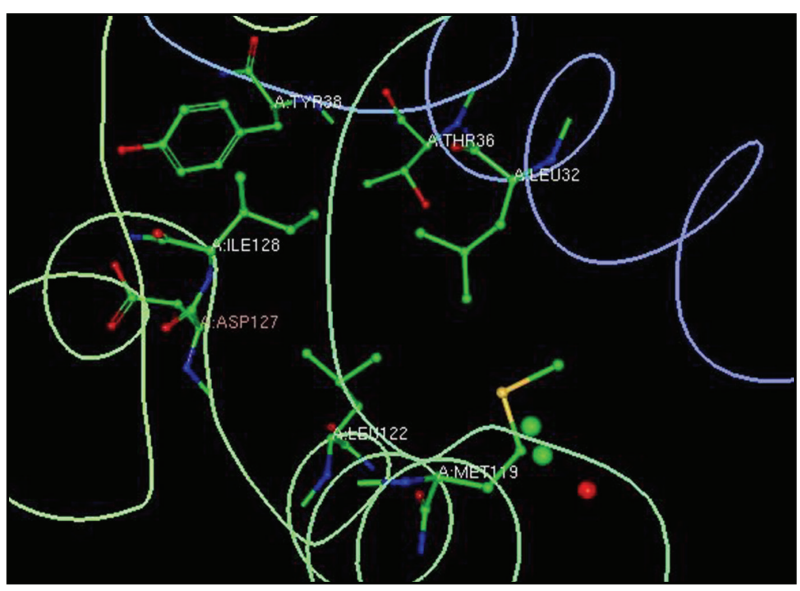

(b)

Figure 2: Molecular visualization of interaction between Heptadecanoic acid, 16 methyl-, methyl ester and the target protein. (a) Proteinligand interaction (Pymol software). (b) Amino acids in the binding pocket (Coil: TYR38, THR36, ILE128, ASP127, MET119); (Alpha: LEU122, LEU32) (RCSB Ligand Explorer).

present study suggested that heptadecanoic acid, 16 methyl, methyl ester could be considered for further in vitro and in vivo studies towards development of ant-skin-cancer drug.

\section{Conclusion}

The present study showed that fatty acid derivative obtained from trichoderma species could be a potent inhibitor against skin cancer protein on the basis of docking scores. We anticipate that further exploration of the functions and molecular mechanisms of the compound will facilitate a better understanding for the control of skin cancer and in development of anticancer drugs.

\section{Acknowledgments}

authors are thankful to the authorities of Annamalai University for providing necessary facilities to carry out this work, and Department of Science \& Technology, Govt. of India, New Delhi.

\section{References}

[1] N. Sithranga Boopathy and K. Kathiresan, "Anticancer drugs from marine flora: an overview," Journal of Oncology, vol. 2010, Article ID 214186, 18 pages, 2010.

[2] S. Ravikumar, M. Gnanadesigan, P. Suganthi, and A. Ramalakshmi, "Antibacterial potential of chosen mangrove plantsagainst isolated urinary tract infectious bacterial pathogens," International Journal of Medicine and Medical Sciences, vol. 2, no. 3, pp. 94-99, 2010.

[3] J. C. Frisvad, T. O. Larsen, R. de Vries et al., "Secondary metabolite profiling, growth profiles and other tools for species recognition and important Aspergillus mycotoxins," Studies in Mycology, vol. 59, no. 1, pp. 31-37, 2007.
[4] J. C. Frisvad, B. Andersen, and U. Thrane, "The use of secondary metabolite profiling in chemotaxonomy of filamentous fungi," Mycological Research, vol. 112, no. 2, pp. 231-240, 2008.

[5] U. Thrane, B. Anderson, J. C. Frisvad, and J. Smedsgaard, "The exo-metabolome in filamentous fungi," in Metabolomics, J. Nielsen and M. C. Jewett, Eds., vol. 18, pp. 235-252, Springer, Berlin, Heidelberg, Germany, 2007.

[6] H. Kusakabe, K. Kodama, H. Machida et al., "Occurrence of a novel enzyme, L-lysine oxidase with antitumor activity in culture extract of Trichoderma viride," Agricultural and Biological Chemistry, vol. 43, no. 2, pp. 337-343, 1979.

[7] H. Kusakabe, K. Kodama, A. Kuninaka et al., "A new antitumor enzyme, L-lysine $\alpha$-oxidase from Trichoderma viride: purification and enzymological properties," The Journal of Biological Chemistry, vol. 255, no. 3, pp. 976-981, 1980.

[8] R. S. Stern, "Prevalence of a history of skin cancer in 2007: results of an incidence-based model," Archives of Dermatology, vol. 146, no. 3, pp. 279-282, 2010.

[9] D. J. Askew and M. D. Laing, "An adapted selective medium for the quantitative isolation of Trichoderma species," Plant Pathology, vol. 42, no. 5, pp. 686-690, 1993.

[10] R. J. Cox, "The biosynthesis of polyketides, acyl tetramic acids and pyridones by filamentous fungi," in Exploitation of Fungi, G. D. Robson, P. van West, and G. M. Gadd, Eds., CUP, 2007.

[11] N. Ruiz, G. Wielgosz-Collin, L. Poirier et al., "New Trichobrachins, 11-residue peptaibols from a marine strain of Trichoderma longibrachiatum," Peptides, vol. 28, no. 7, pp. 1351-1358, 2007.

[12] T. R. A. Thomas, D. P. Kavlekar, and P. A. LokaBharathi, "Marine drugs from sponge-microbe association-a review," Marine Drugs, vol. 8, no. 4, pp. 1417-1468, 2010.

[13] Q. M. Lu, Q. Wei, Y. Jin, J. F. Wei, W. Y. Wang, and Y. L. Xiong, "L-amino acid oxidase from Trimeresurus jerdonii snake venom: purification, characterization, platelet aggregationinducing and antibacterial effects," Journal of Natural Toxins, vol. 11, no. 4, pp. 345-352, 2002.

[14] P. Ciscotto, R. A. Machado de Avila, E. A. F. Coelho et al., "Antigenic, microbicidal and antiparasitic properties of an 
L-amino acid oxidase isolated from Bothrops jararaca snake venom," Toxicon, vol. 53, no. 3, pp. 330-341, 2009.

[15] S. R. Ande, H. Fussi, H. Knauer et al., "Induction of apoptosis in yeast by L-amino acid oxidase from the Malayan pit viper Calloselasma rhodostoma," Yeast, vol. 25, no. 5, pp. 349-357, 2008.

[16] N. Stoppacher, B. Kluger, S. Zeilinger, R. Krska, and R. Schuhmacher, "Identification and profiling of volatile metabolites of the biocontrol fungus Trichoderma atroviride by HS-SPMEGC-MS," Journal of Microbiological Methods, vol. 81, no. 2, pp. 187-193, 2010.

[17] H. Wegele, L. Müller, and J. Buchner, "Hsp70 and Hsp90a relay team for protein folding," Reviews of Physiology, Biochemistry and Pharmacology, vol. 151, pp. 1-44, 2004.

[18] L. Neckers, S. Tsutsumi, and M. Mollapour, "Visualizing the twists and turns of a molecular chaperone," Nature Structural and Molecular Biology, vol. 16, no. 3, pp. 235-236, 2009.

[19] A. Kamal, L. Thao, J. Sensintaffar et al., "A high-affinity conformation of Hsp90 confers tumour selectivity on Hsp90 inhibitors," Nature, vol. 425, no. 6956, pp. 407-410, 2003.

[20] P. A. Brough, W. Aherne, X. Barril et al., "4,5-Diarylisoxazole Hsp90 chaperone inhibitors: potential therapeutic agents for the treatment of cancer," Journal of Medicinal Chemistry, vol. 51, no. 2, pp. 196-218, 2008.

[21] A. Gaikwad, H. Sura, B. Shaphrang, K. Shalini, M. Ray, and K. Kannabiran, "Prediction of interaction between atitumor compouds and target protiens of different cancers by in silico molecular docking studies," Pharmacologyonline, vol. 3, pp. 692-699, 2011. 


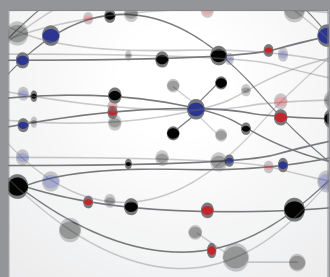

The Scientific World Journal
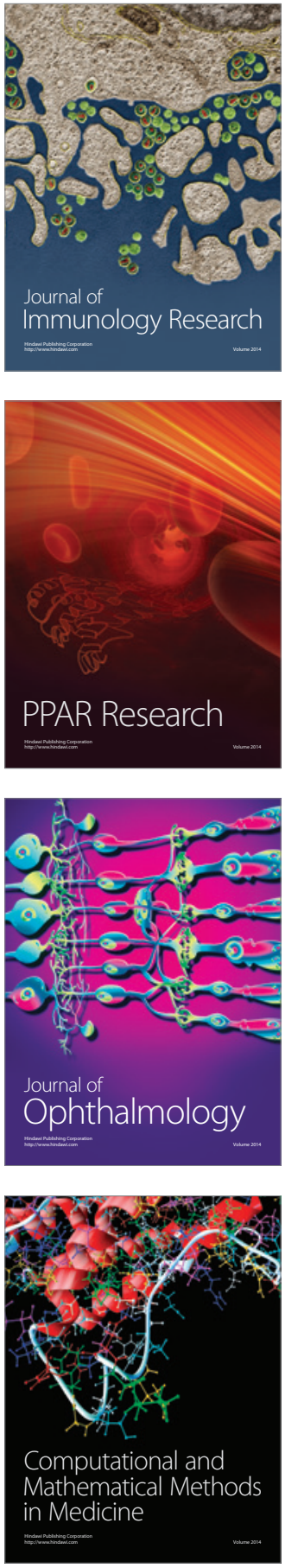

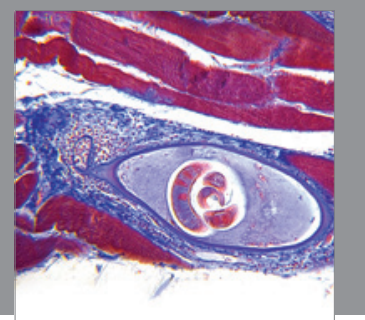

Gastroenterology

Research and Practice
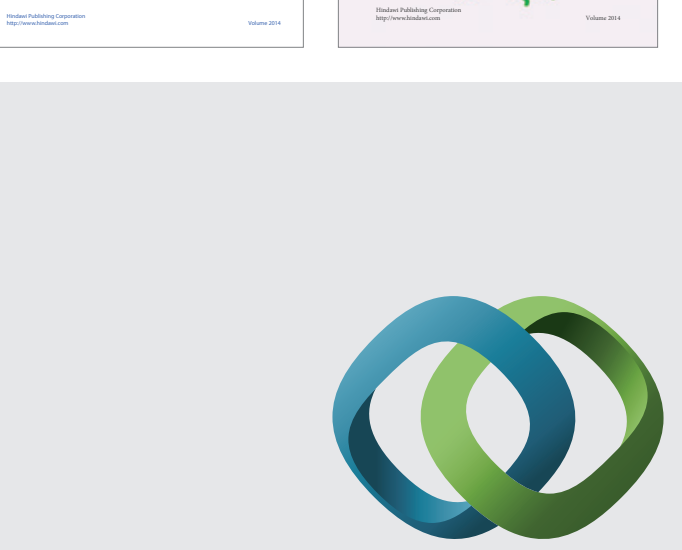

\section{Hindawi}

Submit your manuscripts at

http://www.hindawi.com
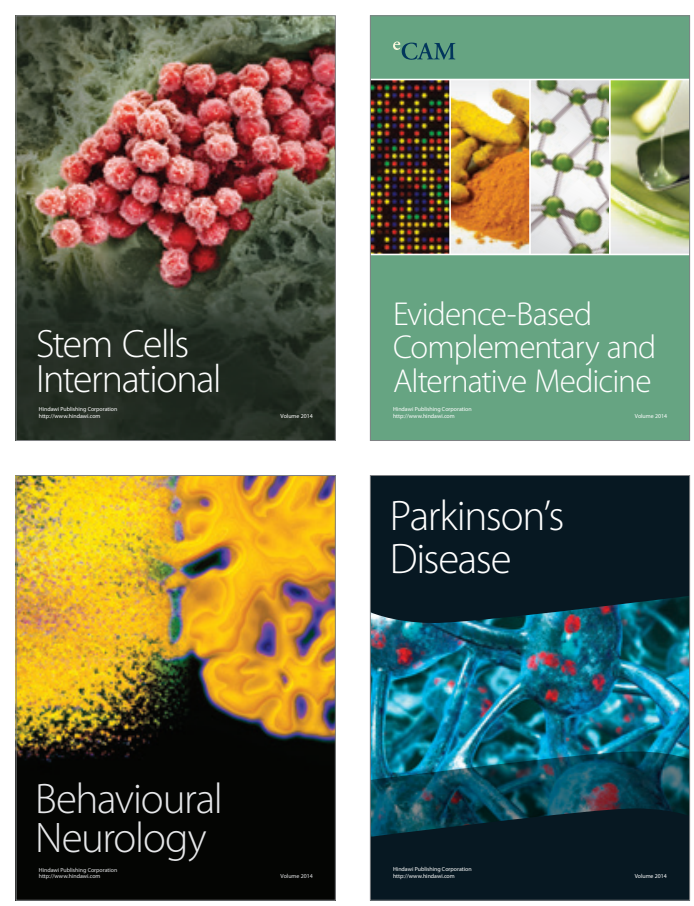

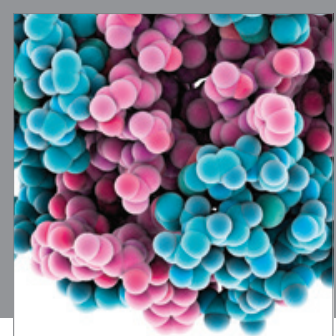

Journal of
Diabetes Research

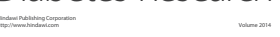

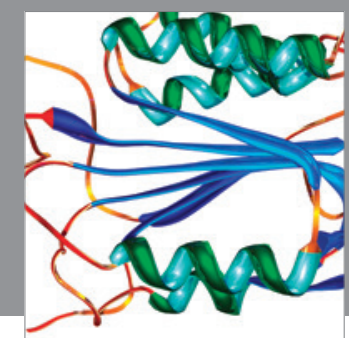

Disease Markers
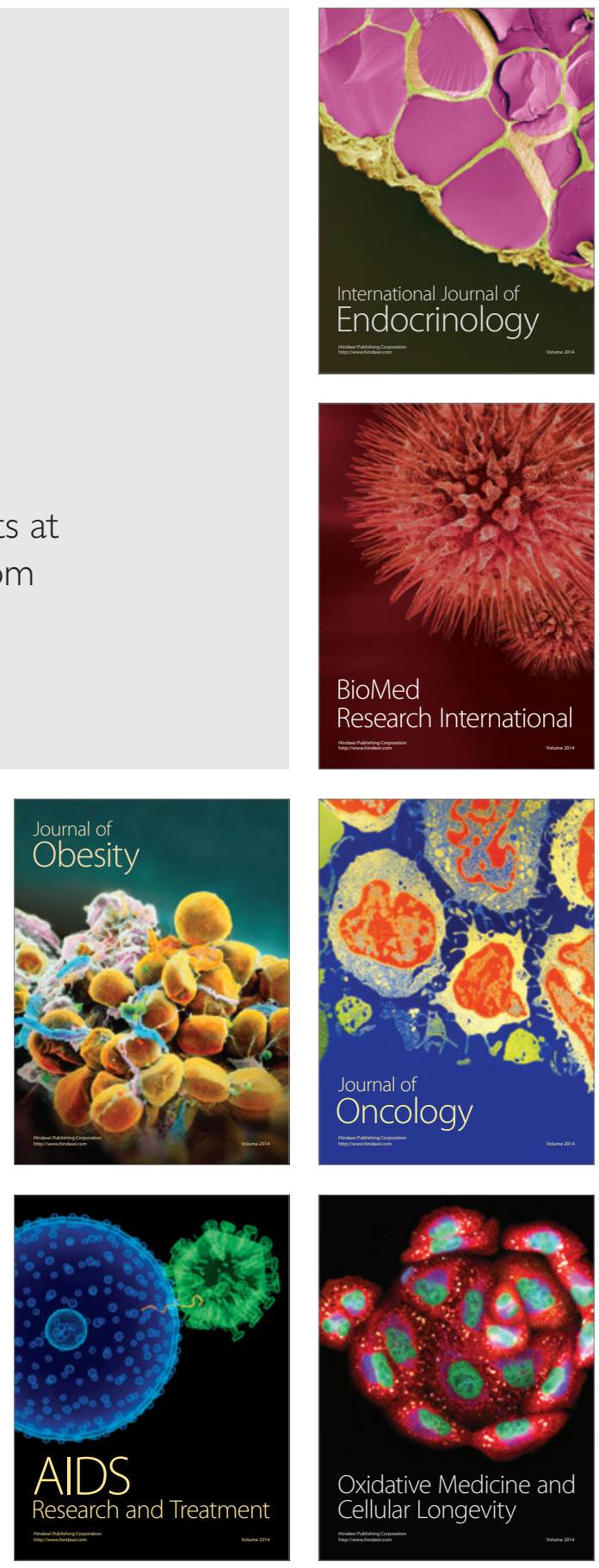